\title{
Living with arthritis: a "training camp" for coping with stressful events? A survey on resilience of arthritis patients following the COVID-19 pandemic
}

\author{
Jacopo Ciaffi ${ }^{1}$ (D) Veronica Brusi ${ }^{1}$ • Lucia Lisi ${ }^{1}$ • Luana Mancarella ${ }^{1}$. Martina D'Onghia ${ }^{1}$ - Elisabetta Quaranta ${ }^{1}$. \\ Antonella Bruni $^{2} \cdot$ Amelia Spinella $^{3} \cdot$ Dilia Giuggioli $^{3} \cdot$ Maria Paola Landini $^{4} \cdot$ Clodoveo Ferri $^{3} \cdot$ Riccardo Meliconi $^{1,5}$. \\ Francesco Ursini ${ }^{1,5}$
}

Received: 22 July 2020 / Revised: 20 August 2020 / Accepted: 16 September 2020 / Published online: 26 September 2020

(C) International League of Associations for Rheumatology (ILAR) 2020

\begin{abstract}
Resilience is defined as "the capacity of individuals to cope successfully with significant change or adversity". The challenge posed by the COVID-19 pandemic may potentially represent an overwhelmingly stressful event for patients with chronic diseases. Aim of our study was to investigate the levels of resilience in individuals with inflammatory arthritis living in Emilia Romagna, the third hardest-hit Italian region during the ongoing COVID-19 pandemic. To this purpose, we developed a survey consisting of four different sections assessing demographic characteristics, the 14-item resilience scale (RS14) and questionnaires evaluating depression and anxiety. Consecutive patients with inflammatory arthritis were recruited over a short time frame immediately after the end of national lockdown and compared with control individuals from the general population. One hundred twenty-two patients and 173 controls were included. Levels of resilience, as measured by RS14 score, were significantly higher in patients with inflammatory arthritis $(82.6 \pm 14.0$ vs $79.0 \pm 12.8, p=0.018)$. After stratification for gender, the difference in RS14 score was maintained in women $(p=0.045)$, but not in men $(p=0.252)$. High resilience, defined as having a RS14 score $>90$, was significantly more prevalent in patients than in controls (30\% vs $16 \%, p=0.009)$. In arthritis patients, no significant differences in RS14 were observed after stratification for specific diagnosis, age, or disease duration and activity. Our findings suggest that patients with inflammatory arthritis may be more resilient than the general population towards unexpected stressful events such as the ongoing COVID-19 pandemic.
\end{abstract}

Key Points

- Living with inflammatory arthritis may foster resilience.

- After COVID-19, patients with inflammatory arthritis were more resilient than the general population.

Keywords Anxiety $\cdot$ Arthritis $\cdot$ COVID-19 $\cdot$ Depression $\cdot$ Resilience

Electronic supplementary material The online version of this article (https://doi.org/10.1007/s10067-020-05411-x) contains supplementary material, which is available to authorized users.

Jacopo Ciaffi

jacopo.ciaffi91@gmail.com

1 Medicine \& Rheumatology Unit, IRCCS Istituto Ortopedico Rizzoli, Bologna, Italy

2 Neuropsychiatric Rehabilitation, Villa Patrizia Hospital, Piossasco, Turin, Italy
3 Rheumatology Unit, University of Modena and Reggio Emilia, Modena, Italy

4 Scientific Direction, IRCCS Istituto Ortopedico Rizzoli, Bologna, Italy

5 Department of Biomedical and Neuromotor Sciences (DIBINEM), University of Bologna, Bologna, Italy 


\section{Introduction}

Several interpretations of the term "resilience" can be retrieved from literature [1], but a widely accepted definition is "the psychological phenomenon representing the capacity of individuals to cope successfully with significant change, adversity or risk" [2]. Adversity is thus the prerequisite, followed by a consequent learning and growing process that, along with a positive adaptation to life's challenges, culminates in the development of resilience [2]. Therefore, resilient individuals are expected to be better equipped to face up to unanticipated changes and stressful situations [2] such as the challenge that humanity has dealt with in the first months of 2020. Indeed, as of August 19, 2020, over 22 million confirmed cases and 780,000 global deaths were reported (https://coronavirus.jhu. edu/map.html) from severe acute respiratory syndrome coronavirus 2 (SARS-CoV-2), the causative agent of the novel coronavirus disease 2019 (COVID-19) [3]. As the infectious threat spread from country to country, unprecedented containment measures were imposed by most national authorities, with a consequent deep economic crisis and compelling social implications [4]. Not unexpectedly, this emergency rebounded immediately on psychological wellness of population, promoting a sudden increase in mental health issues such as depression, anxiety and post-traumatic stress disorder [5, 6]. In this context, an even higher psychological distress may be expected in patients with chronic rheumatic diseases, fuelled by the patients' perception of increased susceptibility to infection arising from the vulnerability associated with the chronic condition itself, the use of immunosuppressive disease-modifying anti-rheumatic drugs (DMARDs) and the difficulty in attending routine visits. On the other side, as suggested by previous research, patients with autoimmune diseases may develop stronger resilience as an adaptive response to the chronicity of their condition $[7,8]$ and be therefore equipped with more effective coping strategies to deal with an unexpected stressful experience such as the ongoing COVID-19 pandemic [9]. On the light of this background, we aimed to investigate the levels of resilience of patients affected by inflammatory arthritis living in Emilia Romagna, the third hardest-hit Italian region during the ongoing COVID-19 pandemic.

\section{Materials and methods}

\section{Design of the study and population}

We conducted a cross-sectional survey from May 11 to June 10, 2020. Target population was composed of consecutive adult patients regularly followed at two academic rheumatology clinics (IRCCS Istituto Ortopedico Rizzoli, Bologna and Policlinico of Modena) located in the Emilia
Romagna region, Italy. To be included in the present study, all patients must have an established diagnosis of rheumatoid arthritis (RA), psoriatic arthritis (PsA) or axial spondyloarthritis (axSpA) fulfilling the appropriate classification criteria [10-12]. The survey was conducted immediately after lockdown measures were eased from Italian Ministry of Health and routine outpatient activity was resumed. Patients were invited to participate to the study at the end of the first follow-up visit after lockdown. A printed copy of the questionnaire was distributed to each patient by the attending physicians after reporting, on a dedicated page, anonymous information regarding disease characteristics. To evaluate disease activity, the ankylosing spondylitis disease activity score with C-reactive protein (ASDAS-CRP) was used in patients with axSpA [13], while the disease activity score including 28 joints and C-reactive protein (DAS28-CRP) was used to homogenize the assessment in RA and PsA $[14,15]$. The stringent DAS28-CRP cut-off of 1.9 was used to define remission, as proposed by previous literature [16]. When the form was completed, patients were asked to close it into a blank envelope to be dropped into a lock box. The lock box was kept closed until the end of the study period to preserve anonymity.

For comparison, a control group from the general population was recruited. The same questionnaire was sent to 500 individuals living in the same geographic area, directly contacted by the members of the research group, using the Google Forms platform (https://docs.google.com/forms/). A unique link to access the online survey page was provided, and participants were explicitly asked to answer the survey only once. Reporting was compliant with the Checklist for Reporting Results of Internet E-survey (CHERRIES) [17].

\section{Survey development and structure}

A group of senior researchers (FU, LL, AB, RM, CF), including a medical psychotherapist (LL) and a psychiatrist (AB), designed the survey draft. Pilot testing was performed on a pool of 20 consecutive patients. Suggestions were collected and the questionnaire was modified accordingly. Finally, the definitive version was reviewed by all study researchers and approved by consensus. The survey consisted of four different sections including a total of 58 questions. Section 1 covered sociodemographic information. In section 2, participants were asked to reply to the 14-item resilience scale (RS14) [18] - a widely used tool for quantitative assessment of resilience - in its validated Italian version [19] provided by Resilience Centre, MT, USA, upon license. Each item is scored on a 7point Likert-type scale from 1 (strongly disagree) to 7 (strongly agree) with a total sum ranging from 14 to 98 . Scores above 90 indicate high resilience, and scores below 56 indicate very low resilience [20].

In section 3, participants replied to the 21 items of the Beck's Depression Inventory II (BDI-II) [21]. The BDI-II is 
a widely used self-administered scale assessing the severity of depression in normal individuals or psychiatric populations. Each item is scored on a 4-point scale ranging from 0 (symptom absent) to 3 (severe symptom) with a total sum ranging from 0 to 63 . A score $\geq 14$ has been considered suggestive of depression [22].

Finally, in section 4, participants had to complete the 14 items of the Hospital Anxiety and Depression Scale (HADS) [23]. The HADS was originally developed to measure anxiety and depression in a general population of medical patients. The questionnaire is composed of 7 questions for anxiety and 7 questions for depression, scored independently (HADS-A and HADS-D, respectively). Each item is scored 0 to 3 , for a total score ranging from 0 to 21 for each domain. A cut-off of $\geq 8$ has been proposed to distinguish between cases and non-cases [24]. Complete structure of the survey is provided in Supplementary Table S1.

\section{Ethical considerations}

The research was conducted in compliance with the Declaration of Helsinki and its latest amendments [25]. No personally identifiable information was collected and data remained completely anonymous throughout the study. The study was approved by the local Ethics Committee (Comitato Etico Area Vasta Emilia Centrale, Bologna, Italy-approval number: 0007795/2020).

\section{Statistical analysis}

Data are expressed as mean \pm standard deviation (SD), median $\left(25^{\text {th }}-75^{\text {th }}\right.$ percentile) or number (percentage) as appropriate. Independent samples $t$ test was used to compare means of continuous variables between two groups. Analysis of covariance (ANCOVA) was conducted to evaluate the level of resilience, depression and anxiety in patients with inflammatory arthritis and controls after adjustment for potential confounders. Age and body mass index (BMI) were included in the model because of significant differences between patients and controls and because, in literature, age and BMI were shown to affect resilience [26-32]. On the other hand, although significant differences in educational level, smoking habit, and presence of chronic comorbidities were also observed, there is no solid literature basis suggesting an influence on resilience. Therefore, with the aim of avoiding model overfitting in consideration of the limited number of patients, we decided to include as potential confounders only covariables known to have an effect on resilience. Analysis of variance (ANOVA) with Bonferroni's correction for multiple comparisons was used to compare resilience between patients with different inflammatory arthritis. For the ANOVA and ANCOVA, statistical assumptions, including normality of data and homogeneity of regression slopes, were assessed, and if assumptions were not met, appropriate transformations were employed. Fisher's exact test was used to compare categorical variables between two groups, and chisquare test was used to compare categorical variables between more than two groups, with post hoc Bonferroni pairwise comparison. Univariate linear regression models were built to evaluate the correlation between continuous variables, expressed as $R^{2}$ coefficient and relative $p$ value. All statistical analyses were performed using the Statistical Package for the Social Sciences (SPSS) software ver. 26.0 (IBM, Armonk, NY, USA).

\section{Results}

\section{General characteristics of the study population}

General characteristics of the study population are reported in Table 1. A total of 122 patients with inflammatory arthritis were included in the study. Of these, $74(61 \%)$ were female, $69(57 \%)$ were affected by RA, 36 (29\%) by PsA and 17 (14\%) by axSpA. Regarding controls, 173 out of 500 individuals replied to the survey. Mean age and BMI were significantly higher in patients than in controls ( $p$ value 0.004 and 0.010 , respectively). Characteristics of patients according to the different disease groups are shown in Supplementary Table S2.

\section{Resilience in patients with rheumatic diseases compared with controls}

In ANCOVA model, patients with inflammatory arthritis showed significantly higher levels of resilience, as measured by RS14, when compared with controls $(82.6 \pm 14.0$ vs $79.0 \pm$ $12.8, p=0.018$; Fig. 1a). Furthermore, levels of depression and anxiety were slightly higher in patients than in controls, although the difference was not significant (BDI-II, 7.4 \pm 7.5 vs $6.0 \pm 5.5, p=0.176$; HADS-D, $5.7 \pm 3.2$ vs $5.2 \pm 2.8, p=$ 0.121 ; HADS-A, $6.5 \pm 3.3$ vs $5.9 \pm 3.5, p=0.184$; Figs. $1 \mathrm{~b}, \mathrm{c}$ and $\mathrm{d}$, respectively). After stratification for gender, the difference in RS14 score was maintained in women $(80.9 \pm 15.8$ vs $77.8 \pm 12.4, p=0.045)$, but not in men $(85.1 \pm 10.4$ vs $81.8 \pm$ 13.5, $p=0.252$ ) (Fig. 1a). The prevalence of high resilience, defined as having a RS14 score $>90$, was significantly higher in patients than in controls ( $30 \%$ vs $16 \%, p=0.009$ ). Using BDI-II cut-off of $\geq 14$ to identify cases of depression, prevalence was $14 \%$ in patients with inflammatory arthritis and $11 \%$ in controls $(p=0.474)$. Using the HADS-D cut-off of $\geq 8,32 \%$ of patients and $18 \%$ of controls $(p=0.008)$ were identified as cases, while the percentages where respectively $40 \%$ and $28 \%(p=0.032)$ when HADS-A was evaluated. 
Table 1 General characteristics of the study population

\begin{tabular}{|c|c|c|c|}
\hline & Patients $(n=122)$ & Controls $(n=173)$ & $p$ value \\
\hline Female, $n(\%)$ & $74(61)$ & $121(70)$ & 0.106 \\
\hline Age, years & $55.7 \pm 15.6$ & $50.9 \pm 12.9$ & 0.004 \\
\hline BMI, $\mathrm{kg} / \mathrm{m}^{2}$ & $25.1 \pm 4.6$ & $23.8 \pm 3.4$ & 0.010 \\
\hline \multicolumn{4}{|l|}{ Educational level } \\
\hline Primary school, $n(\%)$ & $10(8)$ & $6(4)$ & 0.115 \\
\hline Middle school, $n(\%)$ & $31(25)$ & $12(7)$ & $<0.001$ \\
\hline High school, $n(\%)$ & $43(35)$ & $58(33)$ & 0.804 \\
\hline University, $n(\%)$ & $38(32)$ & $97(56)$ & $<0.001$ \\
\hline \multicolumn{4}{|l|}{ Smoking habit } \\
\hline Never smokers, $n(\%)$ & $47(38)$ & $93(54)$ & 0.013 \\
\hline Former smokers, $n(\%)$ & $51(42)$ & $47(27)$ & 0.012 \\
\hline Current smokers, $n(\%)$ & $24(20)$ & $33(19)$ & 1 \\
\hline \multicolumn{4}{|l|}{ Marital status } \\
\hline Married, $n(\%)$ & $75(62)$ & $98(57)$ & 0.472 \\
\hline Single, $n(\%)$ & $30(24)$ & $53(30)$ & 0.294 \\
\hline Divorced, $n(\%)$ & $7(6)$ & $17(10)$ & 0.280 \\
\hline Widowed, $n(\%)$ & $10(8)$ & $5(3)$ & 0.058 \\
\hline $\begin{array}{l}\text { Presence of comorbidities/non-rheumatic } \\
\text { chronic diseases, } n(\%)\end{array}$ & $82(67)$ & $64(37)$ & $<0.001$ \\
\hline \multicolumn{4}{|l|}{ Rheumatic disease } \\
\hline Rheumatoid arthritis, $n(\%)$ & $69(57)$ & & \\
\hline Psoriatic arthritis, $n(\%)$ & $36(29)$ & & \\
\hline Axial spondyloarthritis, $n(\%)$ & $17(14)$ & & \\
\hline Disease duration, years & $10.3 \pm 7.6$ & & \\
\hline DAS28-CRP* post-COVID19 & $2.0 \pm 0.8$ & & \\
\hline ASDAS-CRP** post-COVID19 & $1.4 \pm 0.5$ & & \\
\hline cDMARDs, $n(\%)$ & $72(59)$ & & \\
\hline bDMARDs/tsDMARDs, $n(\%)$ & $56(46)$ & & \\
\hline RS14, score & $82.6 \pm 14.0$ & $79.0 \pm 12.8$ & 0.018 \\
\hline BDI-II, score & $7.4 \pm 7.5$ & $6.0 \pm 5.5$ & 0.176 \\
\hline HADS-D, score & $5.7 \pm 3.2$ & $5.2 \pm 2.8$ & 0.121 \\
\hline HADS-A, score & $6.5 \pm 3.3$ & $5.9 \pm 3.5$ & 0.184 \\
\hline
\end{tabular}

Data are presented as mean \pm standard deviation or number (percentage) as appropriate. For RS14, BDI-II, HADS-D and HADS-A comparison, $p$ values refer to ANCOVA analyses after correction for age and BMI.

Legend: $B M I$ body mass index, $D A S 28-C R P$ disease activity score including 28 joints with $\mathrm{C}$-reactive protein, COVID-19 coronavirus disease 2019, ASDAS-CRP ankylosing spondylitis disease activity score with C-reactive protein, $c D M A R D s$ conventional disease-modifying anti-rheumatic drugs, $B D M A R D s$ biologic disease-modifying anti-rheumatic drugs, tsDMARDs targeted synthetic disease-modifying anti-rheumatic drugs, RS14 14-items resilience scale, $B D I-I I$ Beck's depression inventory version II, HADS-D hospital anxiety depression scale-depression, HADS-A hospital anxiety depression scale-anxiety

*Calculated in patients with rheumatoid arthritis and psoriatic arthritis

**Calculated in patients with axial spondyloarthritis

\section{Resilience in patients with different characteristics}

In ANOVA analysis, resilience levels did not differ significantly between patients with RA, PsA or axSpA (Fig. 2a). After stratification for median age [58.0 years (45.0-65.5)] and disease duration [9.0 years $(4.0-14.3)$ ] we did not observe any significant difference in RS14 score in older vs younger patients $(83.2 \pm 15.2$ vs $82.0 \pm 12.8, p=0.640$, Fig. $2 b)$ or longer vs shorter disease duration $(83.6 \pm 12.6$ vs $81.4 \pm 15.5$, $p=0.390$, Fig. 2c).

Regarding the relationship between disease activity and resilience, we compared RS14 score in RA and PsA patients who achieved remission vs those with active disease. RS14 scores did not significantly differ in patients with inactive vs active disease $(83.7 \pm 13.0$ vs $81.0 \pm 15.5, p=0.349)$ (Fig. $2 \mathrm{~d}$ ). Due to the limited number of patients included, stratification 
a

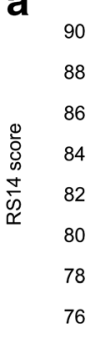

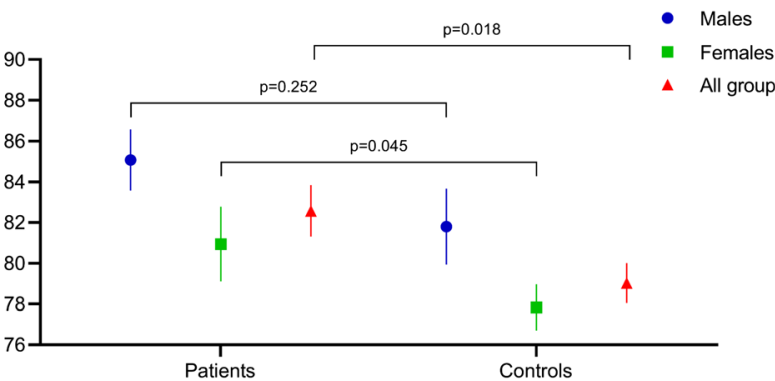

C

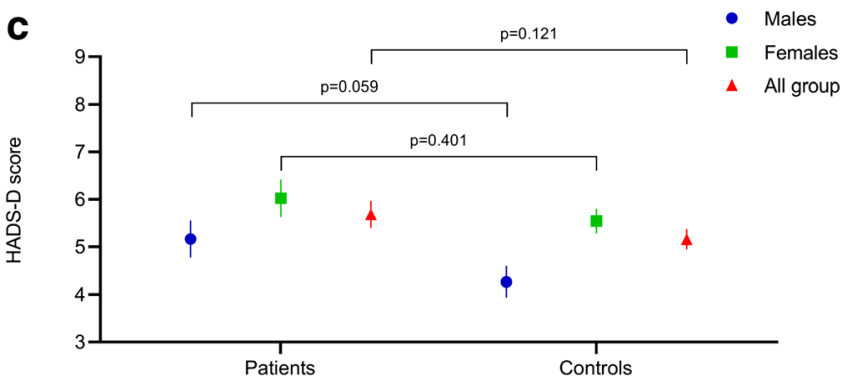

b
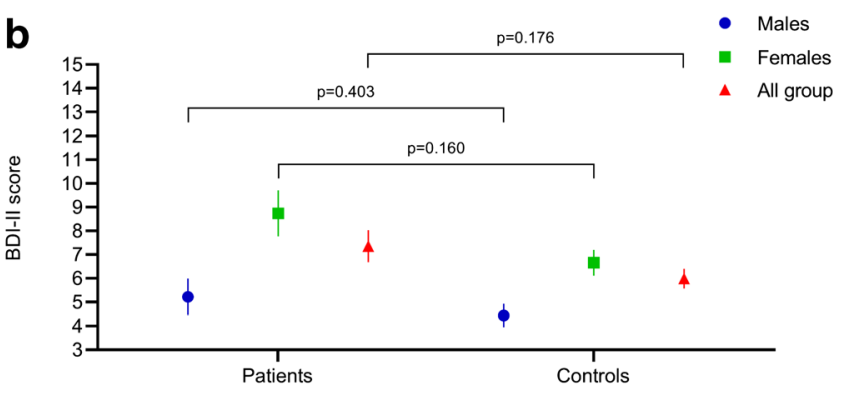

d

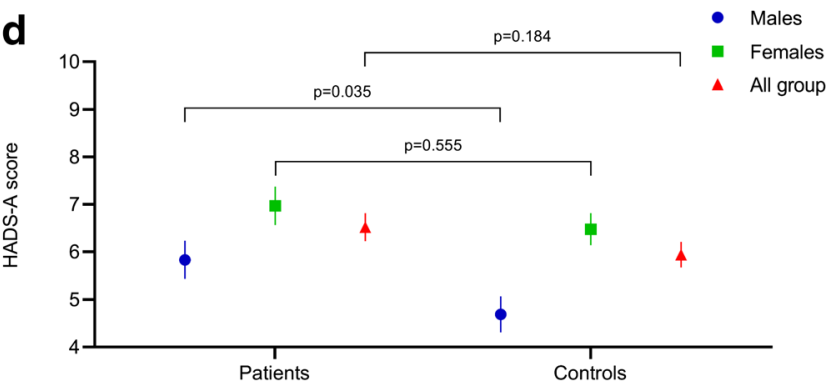

Fig. 1 Resilience (a), depression (b, c) and anxiety (d) in patients with inflammatory arthritis compared with control group. Data are expressed as mean and standard error of the mean and stratified according to gender

for disease activity according to ASDAS-CRP was not possible in individuals with axSpA.

In univariate linear regression analysis, RS14 was inversely correlated with BDI-II score $\left(R^{2}=0.423, p<0.001\right.$; Fig. 3a), HADS-D score $\left(R^{2}=0.725, p<0.001\right.$; Fig. 3b), HADS-A score $\left(R^{2}=0.687, p<0.001\right.$; Fig. $\left.3 \mathrm{c}\right)$ and BMI $\left(R^{2}=0.044, p\right.$ $=0.020$; Fig. 3e) but not with age $\left(R^{2}=0.003, p=0.557\right.$; Fig. $3 \mathrm{D})$ or disease duration $\left(R^{2}=0.001, p=0.720\right.$; Fig. 3f).

a

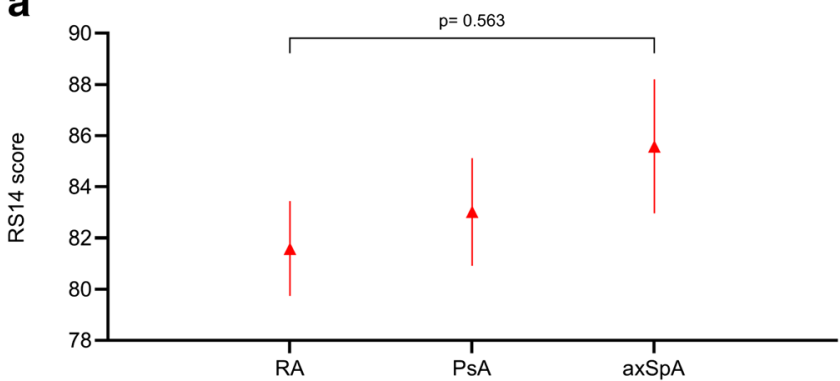

C

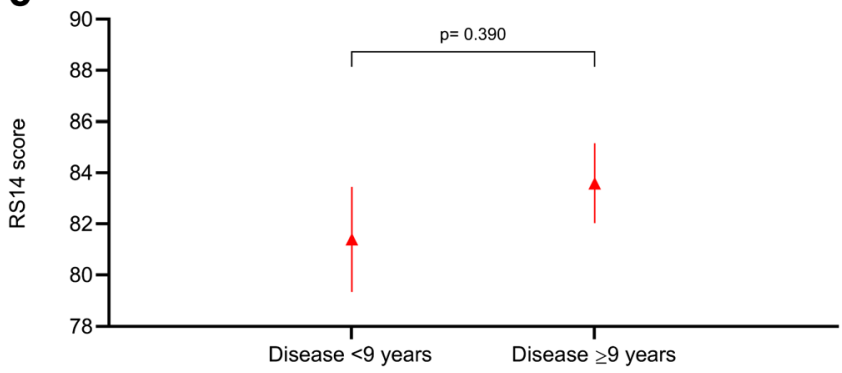

\section{Discussion}

The aim of our study was to investigate levels of resilience in patients with inflammatory arthritis following the COVID-19 outbreak in Italy. Resilience can be defined as a multidimensional construct explaining long-term positive effects derived from the ability to adapt to threatening or traumatic experiences [33]; emerging evidence suggests that a resilient coping

b

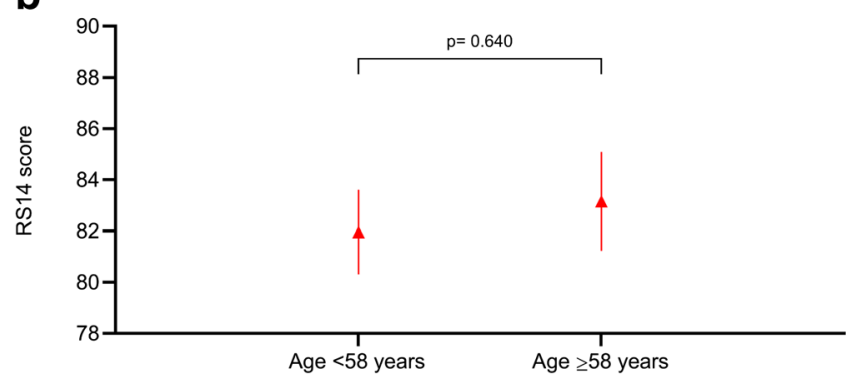

d

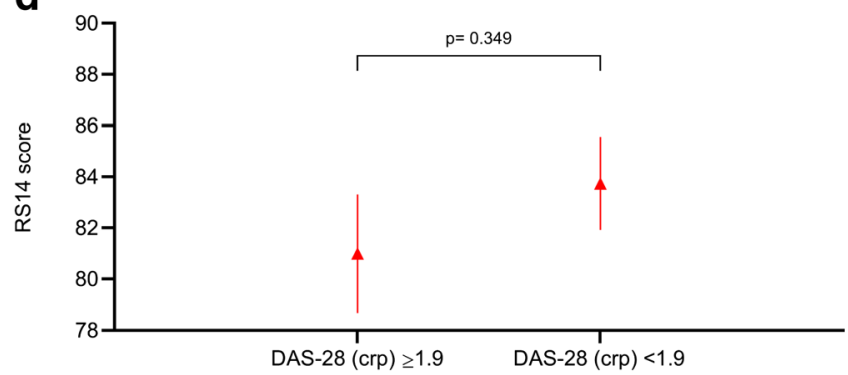

Fig. 2 Levels of resilience in patients with inflammatory arthritis after stratification for diagnosis (a), median age (b), median disease duration (c) and disease activity (d). Data are expressed as mean and standard error of the mean 

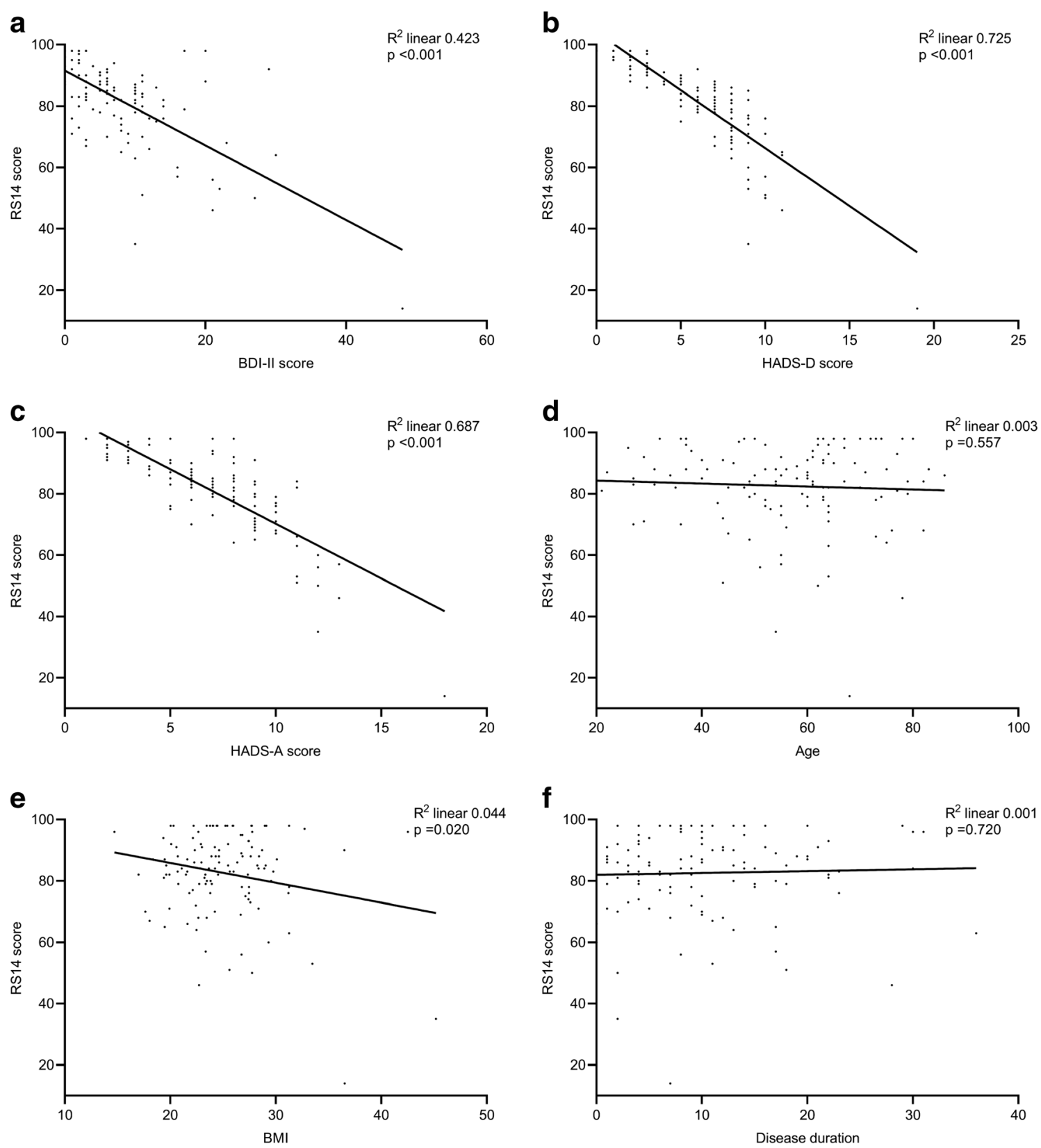

Fig. 3 Linear regression (dot plot with overlayed regression line) showing the association between resilience and depression (a, b), anxiety (c), age (d), body mass index (e) and disease duration (f) in patients with inflammatory arthritis

style may significantly reduce the emotional distress associated with illness experience [34]. Everyday life of patients with rheumatic diseases is often burdened by physical and psychological impairment [8], and different mechanisms are needed to implement cognitive, behavioural, emotional and active coping responses [35] against disease-related stress and challenges. From this perspective, it is therefore conceivable that patients with chronic diseases could be more equipped to face up the strain caused by the COVID-19 pandemic. Our findings preliminarily support this hypothesis. Indeed, levels of resilience obtained in patients with inflammatory arthritis immediately after the first COVID-19 wave were significantly higher than in controls. Interestingly, when comparing levels of resilience in patients with specific disease characteristics, we found no significant differences between younger or older patients, or between cases with longer or shorter disease duration. Thus, according to our data, we can speculate that the development of resilience is a relatively fast adaptive response after a diagnosis of inflammatory arthritis. Furthermore, although underpowered and not reaching the statistical significance, mean RS14 appeared higher in patients with inactive disease, raising the hypothesis that optimal control of the 
inflammatory component may have a role in bolstering resilience of patients with a diagnosis of inflammatory arthritis. Finally, we showed strong, inverse relationship between resilience and symptoms of depression or anxiety, which is expected and in line with clinical experience [36].

Despite providing a novel insight in understanding mechanisms of response to stress in rheumatic patients, our study has some limitations to be acknowledged. First, the design is cross-sectional. As a consequence, we cannot draw conclusions about the short-term change in resilience levels in response to COVID-19 pandemic in arthritis patients. However, if we hypothesize a short-term detrimental effect of pandemic experience on resilience, it remains noteworthy that arthritis patients end up with stronger resilience compared with controls. Secondly, in order to accurately catch the direct effects of COVID-19 experience, we decided to collect data over a very short time frame immediately after restrictive measures were relaxed by health authorities. Unfortunately, although required, this approach prejudiced our ability to recruit a larger cohort, thus preventing the possibility to perform adequately powered sub-analyses on specific rheumatic diseases, or on patients with distinct characteristics. In this view, studies with larger population would be needed to further explore the topic and possibly confirm our results. Moreover, due to the limited number of patients included in our study, we opted to uniform the assessment of disease activity using DAS28-CRP in both RA and PsA with the aim of improving statistical power, although the use of more comprehensive or composite indices would be appropriate in patients with PsA [37]. Another potential limitation is the different modality of requesting to participate to the survey between patients and controls. The former were directly invited by the caring physician at the end of a scheduled follow-up visit, while the latter were contacted through web-based methods. In both cases, the questionnaires were self-administered, and patients were not supervised nor assisted in filling out the survey, but we acknowledge that some information, as for instance comorbidities of controls, may be not completely reliable as there was no mean to verify their accuracy. Finally, for epidemiological reasons, we included only patients with inflammatory arthritis, but we acknowledge that the effects of the pandemic experience on resilience in patients with other connective tissue diseases (e.g. systemic lupus erythematosus) may differ significantly. Therefore, we feel that our results should not be generalized to all patients with inflammatory rheumatic diseases.

In conclusion, our findings suggest that patients with rheumatic diseases might be more resilient than the general population towards unexpected global events generating stress and anxiety. Living with arthritis, despite the well-known difficulties associated with chronic diseases, may represent a "training camp" for developing effective, resilient coping strategies against adversities of life.

\section{Compliance with ethical standards}

The research was conducted in compliance with the Declaration of Helsinki and its latest amendments [25]. No personally identifiable information was collected and data remained completely anonymous throughout the study. The study was approved by the local Ethics Committee (Comitato Etico Area Vasta Emilia Centrale, Bologna, Italy-approval number: 0007795/2020).

Disclosures None

\section{References}

1. Johnston MC, Porteous T, Crilly MA, Burton CD, Elliott A, Iversen L et al (2015) Physical disease and resilient outcomes: a systematic review of resilience definitions and study methods. Psychosomatics. 56(2):168-180

2. Jackson D, Firtko A, Edenborough M (2007) Personal resilience as a strategy for surviving and thriving in the face of workplace adversity: a literature review. J Adv Nurs 60(1):1-9

3. Phelan AL, Katz R, Gostin LO (2020) The novel coronavirus originating in Wuhan, China: challenges for global health governance. JAMA 323(8):709-710. https://doi.org/10.1001/jama.2020.1097

4. Fund $\operatorname{IM}(2020)$ The great lockdown: worst economic downturn since the great depression

5. Gonzalez-Sanguino C, Ausin B, Castellanos MA, Saiz J, LopezGomez A, Ugidos C et al (2020) Mental health consequences during the initial stage of the 2020 coronavirus pandemic (COVID-19) in Spain. Brain Behav Immun 87:172-176. https://doi.org/10.1016/ j.bbi.2020.05.040

6. Wang C, Pan R, Wan X, Tan Y, Xu L, McIntyre RS et al (2020) A longitudinal study on the mental health of general population during the COVID-19 epidemic in China. Brain Behav Immun 87:40 48. https://doi.org/10.1016/j.bbi.2020.04.028

7. Shaw Y, Bradley M, Zhang C, Dominique A, Michaud K, McDonald D et al (2019) The development of resilience among rheumatoid arthritis patients: a qualitative study. Arthritis Care Res (Hoboken) 72(9):1257-1265. https://doi.org/10.1002/acr. 24024

8. Evers AW, Zautra A, Thieme K (2011) Stress and resilience in rheumatic diseases: a review and glimpse into the future. Nat Rev Rheumatol 7(7):409-415

9. Vinkers CH, van Amelsvoort T, Bisson JI, Branchi I, Cryan JF, Domschke $\mathrm{K}$ et al (2020) Stress resilience during the coronavirus pandemic. Eur Neuropsychopharmacol 35:12-16

10. Aletaha D, Neogi T, Silman AJ, Funovits J, Felson DT, Bingham CO 3rd et al (2010) Rheumatoid arthritis classification criteria: an American College of Rheumatology/European League Against Rheumatism collaborative initiative. Arthritis Rheum 62(9):25692581

11. Taylor W, Gladman D, Helliwell P, Marchesoni A, Mease P, Mielants H (2006) Classification criteria for psoriatic arthritis: development of new criteria from a large international study. Arthritis Rheum 54(8):2665-2673

12. Rudwaleit M, van der Heijde D, Landewé R, Listing J, Akkoc N, Brandt J et al (2009) The development of Assessment of SpondyloArthritis international Society classification criteria for axial spondyloarthritis (part II): validation and final selection. Ann Rheum Dis 68(6):777-783

13. Machado PM, Landewé R, Heijde DV (2018) Ankylosing Spondylitis Disease Activity Score (ASDAS): 2018 update of the nomenclature for disease activity states. Ann Rheum Dis 77(10): $1539-1540$ 
14. Wells G, Becker JC, Teng J, Dougados M, Schiff M, Smolen J et al (2009) Validation of the 28-joint Disease Activity Score (DAS28) and European League Against Rheumatism response criteria based on C-reactive protein against disease progression in patients with rheumatoid arthritis, and comparison with the DAS28 based on erythrocyte sedimentation rate. Ann Rheum Dis 68(6):954-960

15. Salaffi F, Ciapetti A, Carotti M, Gasparini S, Gutierrez M (2014) Disease activity in psoriatic arthritis: comparison of the discriminative capacity and construct validity of six composite indices in a real world. Biomed Res Int 2014:528105

16. Fleischmann R, van der Heijde D, Koenig AS, Pedersen R, Szumski A, Marshall L et al (2015) How much does Disease Activity Score in 28 joints ESR and CRP calculations underestimate disease activity compared with the Simplified Disease Activity Index? Ann Rheum Dis 74(6):1132-1137

17. Eysenbach G (2004) Improving the quality of Web surveys: the Checklist for Reporting Results of Internet E-Surveys (CHERRIES). J Med Internet Res 6(3):e34

18. Wagnild G (2009) The resilience scale user's guide for the US English version of the resilience scale and the 14-item resilience scale (RS-14). Resilience Center, Montana, USA

19. Callegari C, Bertu L, Lucano M, Ielmini M, Braggio E, Vender S (2016) Reliability and validity of the Italian version of the 14-item Resilience Scale. Psychol Res Behav Manag 9:277-284

20. Wagnild G (2016) The resilience scale: user's guide for the US English version of the resilience scale and the 14-item resilience scale. Resilience Center, Montana, USA

21. Beck AT, Steer RA, Brown GK (1996) BDI-II: Beck Depression Inventory Manual, 2nd edn. Psychological Corporation, San Antonio

22. Smarr KL, Keefer AL (2011) Measures of depression and depressive symptoms: Beck Depression Inventory-II (BDI-II), Center for Epidemiologic Studies Depression Scale (CES-D), Geriatric Depression Scale (GDS), Hospital Anxiety and Depression Scale (HADS), and Patient Health Questionnaire-9 (PHQ-9). Arthritis Care Res 63(Suppl 11):S454-S466

23. Snaith RPZ(1994) A.S. The Hospital Anxiety and Depression Scale Manual. Nfer-Nelson, Windsor

24. Stern AF (2014) The hospital anxiety and depression scale. Occup Med (Lond) 64(5):393-394

25. World Medical A (2013) World Medical Association Declaration of Helsinki: ethical principles for medical research involving human subjects. JAMA. 310(20):2191-2194

26. Silverman AM, Molton IR, Alschuler KN, Ehde DM, Jensen MP (2015) Resilience predicts functional outcomes in people aging with disability: a longitudinal investigation. Arch Phys Med Rehabil 96(7):1262-1268

27. van Kessel G (2013) The ability of older people to overcome adversity: a review of the resilience concept. Geriatr Nurs 34(2):122127

28. Gooding PA, Hurst A, Johnson J, Tarrier N (2012) Psychological resilience in young and older adults. Int J Geriatr Psychiatry 27(3): 262-270

29. Terrill AL, Molton IR, Ehde DM, Amtmann D, Bombardier CH, Smith AE et al (2016) Resilience, age, and perceived symptoms in persons with long-term physical disabilities. J Health Psychol 21(5):640-649

30. Stewart-Knox B, M ED, Bunting B, Parr H, Vas de Almeida MD, Gibney M (2012) Associations between obesity (BMI and waist circumference) and socio-demographic factors, physical activity, dietary habits, life events, resilience, mood, perceived stress and hopelessness in healthy older Europeans. BMC Public Health 12: 424

31. Wu Z, Liu Y, Li X, Li X (2016) Resilience and associated factors among mainland Chinese women newly diagnosed with breast cancer. PLoS One 11(12):e0167976

32. Nishimi K, Choi KW, Cerutti J, Powers A, Bradley B, Dunn EC (2020) Measures of adult psychological resilience following earlylife adversity: how congruent are different measures? Psychol Med 1-10. https://doi.org/10.1017/S0033291720001191

33. Hornor G (2017) Resilience. J Pediatr Health Care 31(3):384-390

34. Carver CS, Scheier MF, Weintraub JK (1989) Assessing coping strategies: a theoretically based approach. J Pers Soc Psychol 56(2):267-283

35. Bruce TO (2008) Comorbid depression in rheumatoid arthritis: pathophysiology and clinical implications. Curr Psychiatry Rep 10(3):258-264

36. Bavafa A, Khazaie H, Khaledi-Paveh B, Rezaie L (2019) The relationship of severity of symptoms of depression, anxiety, and stress with sleep quality in earthquake survivors in Kermanshah. J Inj Violence Res 11(2):225-232

37. Tucker LJ, Coates LC, Helliwell PS (2019) Assessing disease activity in psoriatic arthritis: a literature review. Rheumatol Ther 6(1): 23-32

Publisher's note Springer Nature remains neutral with regard to jurisdictional claims in published maps and institutional affiliations. 